\title{
Assessment of Micro-Doppler based road targets recognition based on co-operative multi-sensor automotive radar applications.
}

\author{
Pasquale Striano, Christos V. Ilioudis, Carmine Clemente, and John J. Soraghan \\ Centre for Signal and Image Processing, University of Strathclyde, Glasgow, United Kingdom \\ Email: pasquale.striano, c.ilioudis, carmine.clemente, j.soraghan- @ strath.ac.uk
}

\begin{abstract}
Radar systems have become one of the principal sensory components in automotive vehicles, due to their ability to detect and discriminate between different objects in various scenarios. In this paper the micro-Doppler signature is used to identify road targets as cyclist, person, group of people, dog walking, and dog trotting. In order to boost the performance of Automatic Target Recognition in automotive environment, each node could share its micro-Doppler based features in a cooperative manner, using novel Vehicle To Vehicle communication frameworks based on joint radar and communication systems. The classification performance is evaluated considering two scenarios, a single-sensor scenarios where the micro-Doppler signature is observed by a single user, and a multi-sensor scenarios where each user shares its feature vector.
\end{abstract}

Index Terms-ATR, automotive application, single-sensor, multi-sensor

\section{INTRODUCTION}

A typical radar system transmits an electromagnetic wave and receives echo scatterings from a target. When the target is not stationary, it causes a shift in frequency known as the Doppler effect. Additionally, the secondary motions of the target such as limb movement and vibrations may introduce further frequency modulations around the main Doppler component. This phenomenon is well known as the micro-Doppler effect and has been extensively described in [1]. Being caused by secondary motions, micro-Doppler components generally change over time causing a frequency varying signal [1]. These time-frequency modulations represent a unique signature of the target, that allows for algorithms to be developed for Automatic Target Recognition (ATR). The use of microDoppler for ATR has been widely investigated in recent years [2], and it can be now considered a technology ready to be deployed in commercial devices [3].

Radar systems are becoming a key technology for current and future Advanced Driver Assistance Systems (ADAS) due to their capacity to enable a detailed representation of the surroundings, independent of light and weather conditions. Additionally, to increase road security a car could share critical information with other vehicles or infrastructure such as alarms or radar images. In [4] a joint radar communication system based on the Fraction Fourier Transform (FrFT) for automotive applications was presented. The proposed system embeds information in waveforms suitable for radar operations allowing for both communication and sensing to take place simultaneously while meeting low-SWaP (Size, Weight and Power) requirements since no dedicated antenna and transceiver are required for each operation. In [4] it was shown that the radar performance of the proposed waveform design in terms of Ambiguity Function (AF) and Side Lobe Levels is similar to that of a Linear Frequency Modulation (LFM) waveform while also demonstrating that the random nature of the embedded data do not significantly impact the radar performance. Additionally, in [5] it was shown that the proposed framework can work in different communication channels while assuring a good Bit Error Rate (BER).

In this work, the capability to exploit the systems such as the one proposed in [4] in conjunction with micro-Doppler based classification is investigated, with particular emphasis on the possibility to share target information among the different vehicles. Particularly, in single-sensor configuration the classification is based on the observation obtained from a single user, while in multi-sensor each user shares its extracted features with other users. The features are shared using the framework proposed in [4], and they are fused through the Principal Component Analysis (PCA). In the literature, different feature extraction methods for micro-Doppler classification have been proposed including transform-based features [6] and linear predictive coding [7]. In this work the feature vectors are extracted using the Krawtchouk moments due to their benefit of scale, rotation and translation invariant properties [8]. These characteristics, together with the capability to pre-compute the polynomials, make Krawtchouk moments reliable for real time target recognition.

The remaining paper is organized as follows, Section II presents the feature extraction framework from the microDoppler signatures, in single-sensor and multi-sensor scenarios. Section III and Section IV present preliminary classification performance in single-sensor and multi-sensors scenarios respectively. Finally, Section V concludes the paper and discusses future work. 


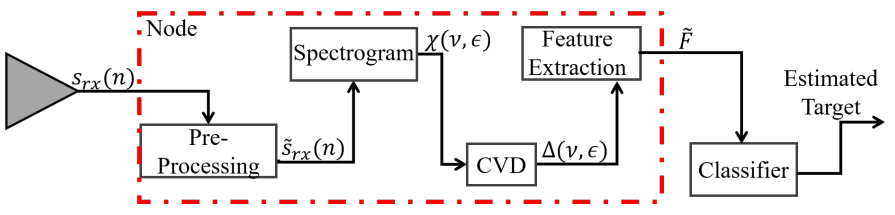

Figure 1: Block diagram of the proposed algorithm for a single-sensor scenarios.

\section{MICRO-DOPPLER CLASSIFICATION}

In this section, the proposed feature vector extraction method using the Krawtchouk moments is presented. The block diagram of the proposed algorithm for a single-sensor scenario is illustrated in Fig. 1. Initially, the algorithm takes as input the received slow time signal $s_{r x}(n)$, with $n=$ $0, \cdots N-1$, containing the micro-Doppler components. At the pre-processing state, $s_{r x}(n)$ is formed into a zero mean and unit variance signal $\tilde{s}_{r x}(n)$. After this operation, to observe the frequency changes over time the Short Time Fourier Transform (STFT) is calculated [9]. Then the spectrogram is obtained through the modulus of the STFT [9]. The spectrogram is a visual representation of the spectrum of the received signal as it varies with time and is expressed as:

$$
\begin{gathered}
\chi(\nu, k)=\left|\sum_{n=0}^{N-1} \tilde{s}_{r x}(n) w_{h}(n-k) e^{-i 2 \nu \frac{n}{N}}\right| \\
k=0, \cdots, K-1
\end{gathered}
$$

where $i=\sqrt{-1}, \nu$ is the normalized frequency and $w_{h}(\cdot)$ is the window function. In the proposed algorithm the spec-



(a) Spectrogram

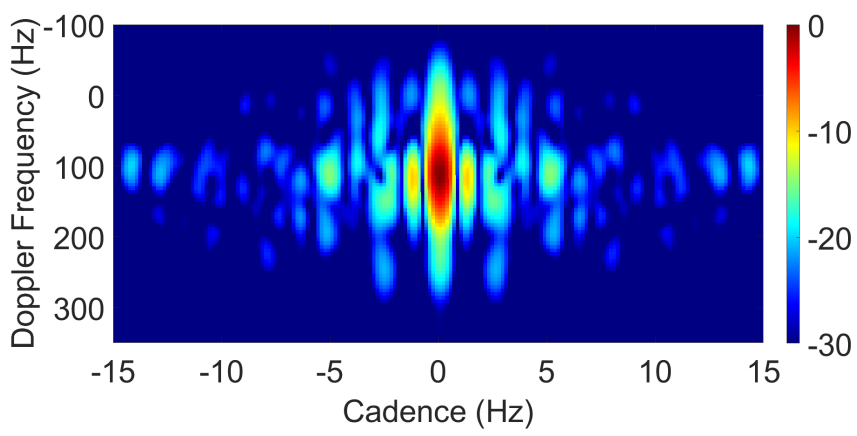

(b) CVD

Figure 2: Example of spectrogram and CVD obtained by a received signal from a person, (a) spectrogram and (b) CVD. trogram is preferred over other time-frequency representations due to its robustness to interference [10] and its low computational complexity. However, the spectrogram as defined in (1) will depend on the initial motion phase that the target is when captured. This means that different signatures will be obtained for the same motion if the target is captured with a different initial motion phase. To avoid this issue the Cadence Velocity Diagram (CVD) is used. The CVD is defined as the Fourier Transform of the spectrogram along each frequency bin [11], which can be written as:

$$
\Delta(\nu, \epsilon)=\left|\sum_{k=0}^{K-1} \chi(\nu, k) e^{-i 2 \nu \frac{k}{K}}\right|
$$

where $\epsilon$ is the cadence frequency. The CVD is chosen due its ability to extract useful information such as the cadence of each frequency component and the maximum Doppler shift which are invariant to the initial phase of the motion. To obtain a matrix whose values belong to the set $[0,1]$ the magnitude of the CVD obtained by (2) is normalized as follows

$$
\bar{\Delta}(\nu, \epsilon)=\frac{\Delta(\nu, \epsilon)-\min _{\nu, \epsilon} \Delta(\nu, \epsilon)}{\max _{\nu, \epsilon}\left[\Delta(\nu, \epsilon)-\min _{\nu, \epsilon} \Delta(\nu, \epsilon)\right]}
$$

After extracting the CVD, the feature extraction function is applied. The feature vector, $\mathbf{F}=\left[F_{0}, F_{1}, \cdots, F_{Q-1}\right]$, of dimension $Q$, which is desired to identify unequivocally each class, is obtained using the Krawtchouk moments as well described in [12] and [13]. In order to avoid that polarized vector may affect the classification performance, the feature vector is normalised as follows:

$$
\tilde{\mathbf{F}}=\frac{\mathbf{F}-\eta_{\mathbf{F}}}{\sigma_{\mathbf{F}}}
$$

where $\eta_{\mathbf{F}}$ and $\sigma_{\mathbf{F}}$ are, respectively, the mean and standard deviation of $\mathbf{F}$. The Krawtchouk moments are suitable for this application especially due to their scale invariant property. Fig. 2(a) shows a spectrogram obtained from the simulated signal from a person. As it can be seen the spetrogram depends on the initial phase of the motion and the number of the period depends on the length of the signal. On the other hand, the corresponding CVD is shown in Fig. 2(b). The CVD is characterized by vertical lines whose the position depend on the micro-motions. In Fig. 2(b) the line at zero cadence is the main Doppler where the periodicity is zero, while the

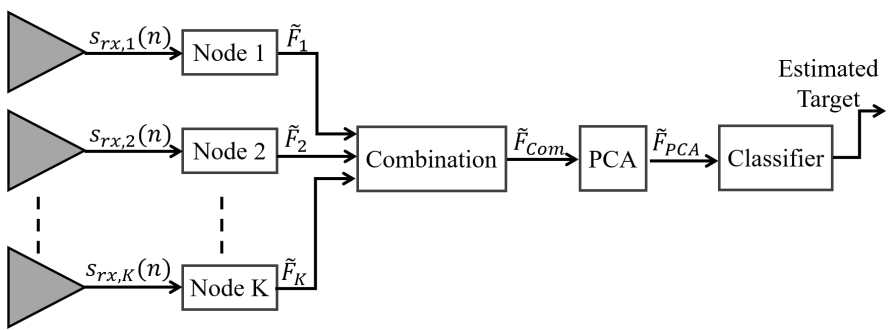

Figure 3: Block diagram of the proposed algorithm in multisensor scenarios. 
second line at $1.36 \mathrm{~Hz}$ means that a micro-motion component with periodicity of 0.7 seconds is present. The application of Krawtchouk moments is chosen as an efficient way to extract local characteristics from the CVD image [14].

The proposed block diagram to extract the feature vector when $K$ sensors are present is illustrated in Fig. 3. In this scenario, each user receivers $K$ feature vectors extracted using the single sensor algorithm. These vectors are combined into a new feature vector, $\tilde{F}_{C o m}$, of $K Q$ elements. After this stage, the PCA is applied on $\tilde{F}_{C o m}$. The PCA, [15], is a statistical procedure that uses an orthogonal transformation to convert the combined feature vector of $K Q$ elements into a new feature vector $\tilde{F}_{P C A}$ that contains $M$ uncorrelated elements.

The classification performances of the extracted feature vectors, in both scenarios, are evaluated using a k-Nearest Neighbour (kNN) classifier for its low complexity.

\section{Classification Performance considering a SINGLE-SENSOR SCENARIO}

In this section, the classification performance is evaluated in a single-sensor scenario considering three main classes of road targets. The examined main targets are human, cyclist and dog (German Shepherd). The simulated targets are shown in Fig. 4. Additionally, the dog class is made up of dog trotting and dog walking classes while the human class consist of other two classes as single person and group of people.

For the human class the model shown in Fig.4(a) is used, while the kinematic and radar return model described in [1] are implemented. The dog kinematic model is based on the data captured for healty German Shepherd [16] and its physical model is shown in Fig.4(b). The differences between dog

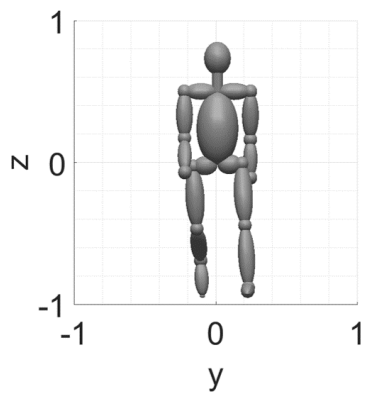

(a)

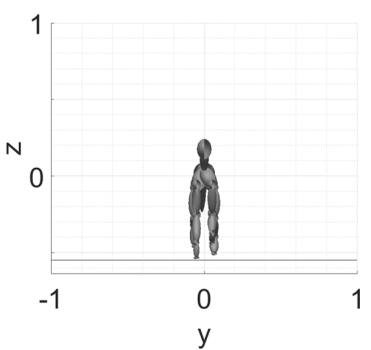

(b)

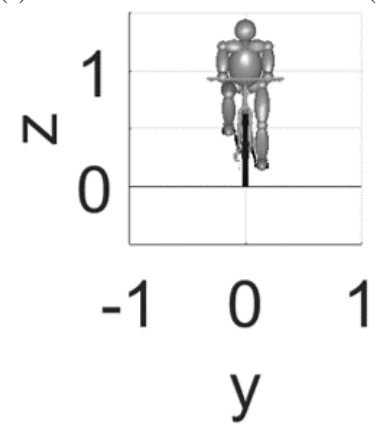

(c)

Figure 4: Target, (a) person, (b) dog, (c) bike.

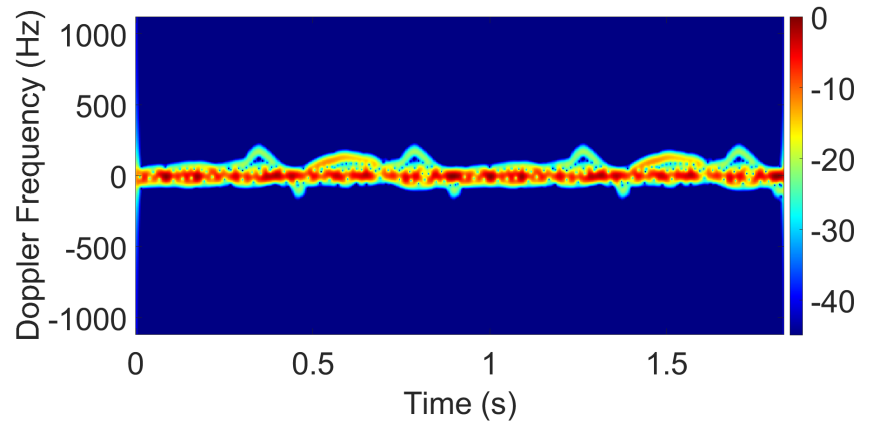

(a) Dog

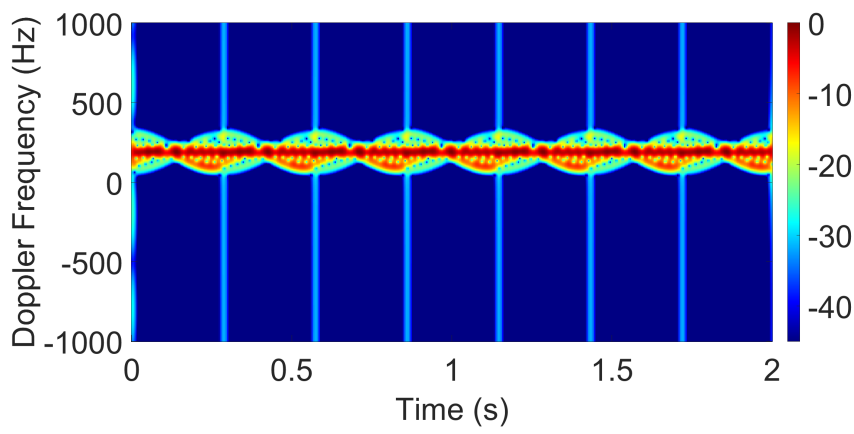

(b) Cyclist

Figure 5: Simulated spectrograms, (a) dog, and (b) cyclist.

walking and dog trotting are their velocity. Finally, the model of a cyclist on a bike applies the forward movement and the rotation of the wheels, of the pedals arms and the partial rotation of the pedals with the physical model show in Fig.4(c). As the micro-Doppler depends on the motion of the target, each target has a different signature. Fig. 2(a) shows the simulated micro-Doppler of a single person, while the simulated spectrograms obtained when the signal is scattered by a dog and cycle are, respectively, shown in Fig. 5. Comparing the spectrograms obtained from these three targets, we can see that for a fixed time duration the number of cycles of motion (signature periodicity) depends on the target. Moreover, the micro-Doppler is around the main Doppler component which depends on the target's main velocity.

The system's performance is evaluated in terms of the Accuracy $\left(\mathrm{A}_{\mathrm{CC}}\right)$ against different values of the Signal Noise Ratio (SNR) and confusion matrices. The accuracy is the rate of correctly classified targets over the entire test set. The noise is added on the CVD before the normalization and is modelled as Additive White Gaussian Noise (AWGN) with $\mathrm{SNR} \in[-5,15] \mathrm{dB}$. The feature vector is extracted as described in Section II, see Fig. 1 and in this analysis the order of the Krawthouck moments is set at 3, while a 1-Nearest Neighbor classifier is used.

In this analysis three classifiers are applied as illustrated in Fig. 6. The first classifier, labeled with CF1, is applied to discriminate between the tree main classes (human, dog and cyclist). While the second and third, labeled with CF21 and $\mathrm{CF} 22$, are used to discriminate the sub-classes that comprise the main classes. In particular the classifier CF21 is used to 
Table I: Interval of parameters.

\begin{tabular}{|l|l|l|}
\hline \multicolumn{3}{|c|}{} \\
\hline target & height $(\mathrm{m})$ & velocity \\
\hline person & {$[1.6,1.8]$} & {$[1,2.5] \mathrm{m} / \mathrm{s}$} \\
\hline cyclist & {$[1.4,1.6]$} & {$[8,16] \mathrm{km} / \mathrm{h}$} \\
\hline dog walking & {$[0.52,0.62]$} & {$[1,3.5] \mathrm{m} / \mathrm{s}$} \\
\hline dog trot & {$[0.55,0.65]$} & $3.5,5 \mathrm{~m} / \mathrm{s}$ \\
\hline group of $\mathrm{p}$ & {$[1.55,1.75]$} & {$[0.5,2.5] \mathrm{m} / \mathrm{s}$} \\
\hline
\end{tabular}

discriminate between a single person and group of people, while the classifier CF22 is implemented to discriminate the action of the dog walking and dog trotting.

Each target is observed considering 36 different azimuth angles. These angles are distributed uniformly between 0 to $2 \pi$. Moreover, for each angle the same target is taken into account 5 times with different height and velocity parameters. This means that for the same class of the target 180 observations have been acquired. The values of the heights and velocities are chosen in random uniformly manner where the perspective interval are listed in Table I.

The duration of the signal used to extract the CVD is 4 seconds while the Pulse Repetition Frequency (PRF) is $1.5 \mathrm{kHz}$. The carrier frequency of the system is $f_{c}=77 \mathrm{GHz}$ while the range resolution is 1 meters. Additionally, each signal starts with a different motion phase. The performance is computed over 500 Monte Carlo runs for each SNR, and for each iteration the observations are divided randomly in $60 \%$ used to train the classifier and $40 \%$ for testing. The differences between the observation used for testing and training are the different noise and the azimuth angel. Where the azimuth angle are chosen in a random manner.

In Fig. 7, the Accuracy obtained, from the classifier CF1, considering the three main classes (human, dog and cyclist) is illustrated. Fig. 7 shows that when SNR $\in[-5,15] \mathrm{dB}$ the Accuracy moves from 0.96 to 0.976 . This means that even for low values of SNR the system is capability to discriminate the three main classes. An example of confusion matrix when

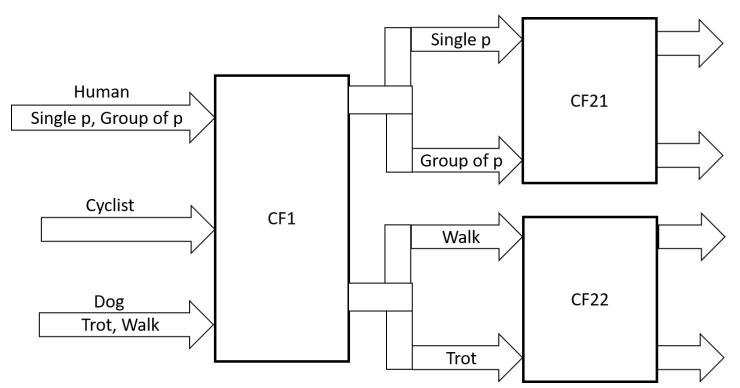

Figure 6: Classifier design.

Table II: Confusion matrix of the classifier CF11 SNR $=-5$ $\mathrm{dB}$, single-sensor scenario.

\begin{tabular}{|l|l|l|l|}
\hline \multicolumn{4}{|c|}{ ATR } \\
\hline Truth & human & dog & cyclist \\
\hline human & $94.1 \%$ & $5.1 \%$ & 0.8 \\
\hline dog & $2.15 \%$ & $97.85 \%$ & 0 \\
\hline cyclist & $3.3 \%$ & $0 \%$ & 96.7 \\
\hline
\end{tabular}

Table III: Confusion matrix of the classifier $\mathrm{CF} 21 \mathrm{SNR}=-5$ $\mathrm{dB}$, single-sensor scenario.

\begin{tabular}{|l|l|l|}
\hline \multicolumn{3}{|c|}{ ATR } \\
\hline Truth & single person & group of people \\
\hline single person & $77.1 \%$ & $22.9 \%$ \\
\hline group of people & $26.8 \%$ & $73.2 \%$ \\
\hline
\end{tabular}

Table IV: Confusion matrix of the classifier CF21 SNR= 15 $\mathrm{dB}$, single-sensor scenario.

\begin{tabular}{|l|l|l|}
\hline \multicolumn{3}{|c|}{ ATR } \\
\hline Truth & single person & group of people \\
\hline single person & $78.9 \%$ & $21.1 \%$ \\
\hline group of people & $24.4 \%$ & $75.6 \%$ \\
\hline
\end{tabular}

SNR is at $-5 \mathrm{~dB}$ is given in Table III. This result suggests that the proposed system can be applied in environment where low SNR values may be presented, as an automotive environment.

The Accuracy obtained from the classifier CF21 and CF22 is illustrated in Fig. 8. As it can be seen when the classifier CF21 is applied the Accuracy moves from 0.75 to 0.77 . Additionally, the Accuracy obtained from the classifier CF22 moves from 0.6 to 0.7. Comparing these two Accuracy's, we can see that the activity of the human classes is better classified than the dog activity.

For a better understating of these results the confusion matrices considering the human classes, classifier CF21, when SNR is set at $-5 \mathrm{~dB}$ and $15 \mathrm{~dB}$, are given in Table III and Table IV, respectively. Under closer inspection, it can be seen that for high SNR values a person and group of people are detected only the $78.9 \%$ and $75.06 \%$ of the times, respectively. Additionally, the confusion mateices obtained from the classifier CF22 when SNR is at $-5 \mathrm{~dB}$ and $15 \mathrm{~dB}$, are given in Table V and Table VI. When SNR is set at $-5 \mathrm{~dB}$ the missclassification of the dog trotting and dog walking are 0.4 and 0.38 , while when SNR moves from $-5 \mathrm{~dB}$ to $15 \mathrm{~dB}$ the missclaffication decreased by 0.293 and 0.1 , respectively. In this section it has been shown that the first classifier is capability to distinguish between the three main classes, and the performance does not depend highly on SNR. Additionally, when second stage classifiers are used to discriminate sub-classes of the main class, high values of missclafficiation are obtained.

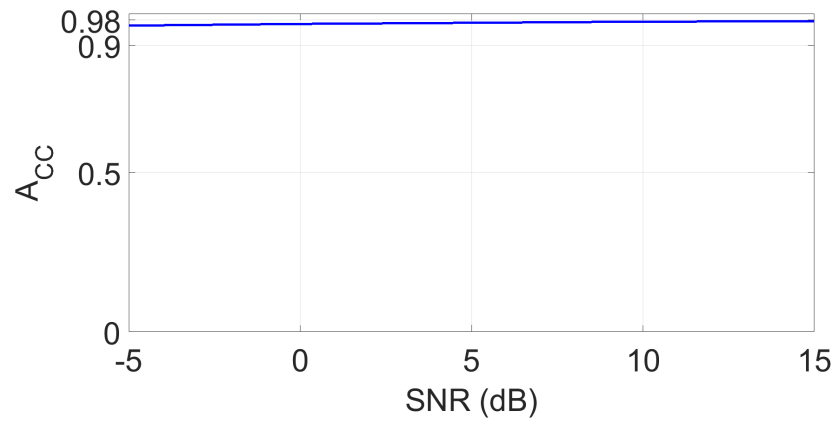

Figure 7: Accuracy obtained from the first classifier, for different SNR values, considering a single-sensor scenario. 


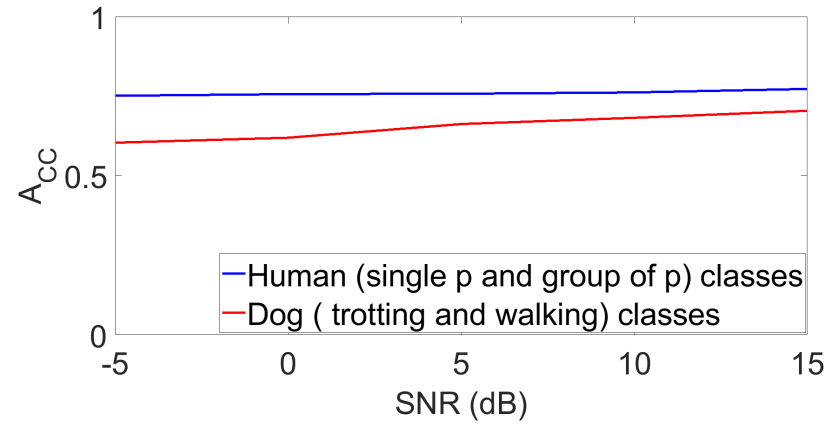

Figure 8: Accuracy obtained from the classifier CF21 and CF22 for different SNR values, considering a single-sensor scenario.

Table V: Confusion matrix of the classifier CF22 SNR $=-5 \mathrm{~dB}$, single-sensor scenario.

\begin{tabular}{|l|l|l|}
\hline \multicolumn{3}{|c|}{ ATR } \\
\hline Truth & dog walking & dog trotting \\
\hline dog walking & $61.2 \%$ & $38.8 \%$ \\
\hline dog trotting & $40.43 \%$ & $59.57 \%$ \\
\hline
\end{tabular}

Table VI: Confusion matrix of the classifier CF22 SNR=15 $\mathrm{dB}$, single-sensor scenario.

\begin{tabular}{|l|l|l|}
\hline \multicolumn{3}{|c|}{ ATR } \\
\hline Truth & dog walking & dog trotting \\
\hline dog walking & $71.02 \%$ & $28.98 \%$ \\
\hline dog trotting & $69.7 \%$ & $30.3 \%$ \\
\hline
\end{tabular}

\section{Classification Performance CONSIDERING A MULTI-SENSOR SCENARIO}

In an automotive environment where more than one user shares the same channel at the same time, low values of SNR might be experienced. As discussed in Section III, in a single-sensor scenario, the system might not be adequate to distinguish between the behaviour of the same target. In order to improve the classification performance each user could use a joint radar communication system, as the one proposed in [4], to share its feature vector with other users. In this section, the performance is evaluated when three sensors are present. The feature vector is extracted as described in Section II. The number of uncorrelated elements $M$ is chose such that the sum of the percentage of the total variance explained by each principal component is more than $75 \%$ [15]. The Krawthchouk moments and the kNN classifier parameters are the ones used in Section III. The performance is evaluated over 500 Monte Carlo runs for each value of SNR. Moreover, for each iteration, the observations obtained from the three users

Table VII: Confusion matrix of the classifier $\mathrm{CF} 1 \mathrm{SNR}=-5$ $\mathrm{dB}$, multiple-sensor scenario.

\begin{tabular}{|l|l|l|l|}
\hline \multicolumn{4}{|c|}{ ATR } \\
\hline Truth & human & dog & cyclist \\
\hline human & $99.94 \%$ & $0 \%$ & 0.06 \\
\hline dog & $0 \%$ & $100 \%$ & 0 \\
\hline cyclist & $0.07 \%$ & $0 \%$ & 99.93 \\
\hline
\end{tabular}

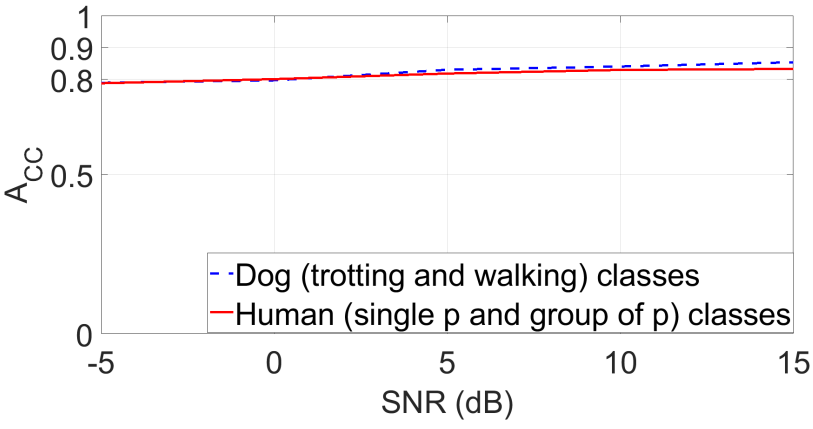

Figure 9: Accuracy obtained from the classifiers CF21 and CF22 for different SNR values, considering a multi-sensor scenario.

are fused in a random manner. At this point, for each iteration, the $60 \%$ of the observations are used for the training while the $40 \%$ for the testing. Furthermore, in each iteration, the PCA is applied on the training data and then transformation is applied on the test data.

The Accuracy obtained from the classifier CF1 for each SNR values is 0.99. An example of the confusion matrix when SNR is at $-5 \mathrm{~dB}$ is given in Table VII. Comparing the Accuracy obtained considering a single-user (Section III) with multiple-user scenarios, it can be seen that when SNR is at $-5 \mathrm{~dB}$ the Accuracy is increased by 0.039 , while when SNR is at $15 \mathrm{~dB}$ the increased is by 0.029 .

The Accuracy obtained from the classifiers CF21 and CF22 is illustrated in Fig. 9. In this scenario, the Accuracy obtained form the CF2 1 moves from 0.78 to 0.82 , while those obtained considering the classifier CF22 moves from to 0.780 .85 . Consequently both classifiers CF21 and CF22 are capability to discriminate the activity of the main class.

Comparing the results obtained considering a single-sensor with the multi-sensor analysis, we can see that when three users share their feature vectors the system is capable to distinguish sub-classes of classes even in environment with low SNR values.

\section{CONCLUSiON}

In this paper a novel micro-Doppler based road targets recognition scheme was proposed using joint radar and communication operation to enable co-operative multi-static observations. The performance has been evaluated in both singlesensor and multi-sensor scenarios. In a single-sensor scenario, the classification is based on the micro-Doppler obtained by a single user, while in multi-sensor scenario each user shares its information with other users and the feature vector are focused applying the PCA. The results demonstrated that in automotive environment, the target recognition based on a co-operative multi-sensor are significantly better than the single-sensor. Future analysis include micro-Doppler signature extraction in multi-static radar scenarios, and experimental validation of the proposed system. 


\section{ACKNOWLEDGMENT}

This work is supported by NXP Laboratories UK Limited.

\section{REFERENCES}

[1] V. Chen, The micro-Doppler Effect in Radar, 1st ed. Artech House, 2011.

[2] C. Clemente, A. Balleri, K. Woosbridge, and J. J. Soraghan, "Developments in target micro-doppler signatures analysis: radar imaging, ultrasound and through-the-wall radar," EURASIP Journal on Advances in Signal Processing, 2013.

[3] L. Pallotta, C. Clemente, A. De Maio, J. J. Soraghan, and A. Farina, "Pseudo-zernike moments based radar micro-doppler classification," in 2014 IEEE Radar Conference, pp. 0850-0854, May 2014.

[4] P. Striano, C. V. Ilioudis, C. Clemente, and J. J. Soraghan, "Communicating radar using frequency-shift keying and fractional fourier transform for automotive applications," The Journal of Engineering, vol. 2019, no. 19, pp. 6016-6020, 2019.

[5] P. Striano, C. V. Ilioudis, C. Clemente, and J. J. Soraghan, "Performance of a communicating radar using fsk and fractional fourier transform for automotive applications," in 2019 IEEE Radio and Wireless Symposium (RWS), pp. 1-4, Jan 2019.

[6] H. Baali, A. Khorshidtalab, M. Mesbah, and M. J. E. Salami, "A transform-based feature extraction approach for motor imagery tasks classification," IEEE Journal of Translational Engineering in Health and Medicine, vol. 3, pp. 1-8, 2015.

[7] Z. Lv, X. Wu, and M. Li, "A research on eog feature parameters extraction based on linear predictive coding model," in 2009 3rd International Conference on Bioinformatics and Biomedical Engineering, pp. 1-4, 2009.

[8] P. . Yap, R. Paramesran, and Seng-Huat Ong, "Image analysis by krawtchouk moments," IEEE Transactions on Image Processing, vol. 12, no. 11, pp. 1367-1377, 2003.

[9] V. C. Chen, F. Li, S. . Ho, and H. Wechsler, "Micro-doppler effect in radar: phenomenon, model, and simulation study," IEEE Transactions on Aerospace and Electronic Systems, vol. 42, no. 1, pp. 2-21, 2006.

[10] A. R. Persico, C. Clemente, C. Ilioudis, D. Gaglione, J. Cao, and J. Soraghan, "Micro-doppler based recognition of ballistic targets using 2d gabor filters," in 2015 Sensor Signal Processing for Defence (SSPD), pp. 1-5, Sep. 2015.

[11] L. Pallotta, C. Clemente, A. De Maio, J. J. Soraghan, and A. Farina, "Pseudo-zernike moments based radar micro-doppler classification," in 2014 IEEE Radar Conference, pp. 0850-0854, May 2014.

[12] C. Clemente, L. Pallotta, D. Gaglione, A. De Maio, and J. J. Soraghan, "Automatic target recognition of military vehicles with krawtchouk moments," IEEE Transactions on Aerospace and Electronic Systems, vol. 53, pp. 493-500, Feb 2017.

[13] A. R. Persico, C. Clemente, L. Pallotta, A. De Maio, and J. Soraghan, "Micro-doppler classification of ballistic threats using krawtchouk moments," in 2016 IEEE Radar Conference (RadarConf), pp. 1-6, May 2016.

[14] A. Sit and D. Kihara, "Comparison of image patches using local moment invariants," IEEE Transactions on Image Processing, vol. 23, pp. 2369 2379, May 2014.

[15] Jolliffe, I. T. Principal Component Analysis. 2nd ed. Springer, 2002.

[16] N. Miqueleto, S. Rahal, F. Agostinho, E. Siqueira, F. Araújo, and A. El-Warrak, "Kinematic analysis in healthy and hip-dysplastic german shepherd dogs," The Veterinary Journal 195.2, 2013. 